\title{
Steering of a Bosonic Mode with a Double Quantum Dot
}

\author{
T. Brandes, N. Lambert \\ Department of Physics, University of Manchester Institute of Science and Technology (UMIST), \\ P.O Box 88, Manchester M60 1QD, United Kingdom
}

(Dated: June 11, 2018)

\begin{abstract}
We investigate the transport and coherence properties of a double quantum dot coupled to a single damped boson mode. Our numerically results reveal how the properties of the boson distribution can be steered by altering parameters of the electronic system such as the energy difference between the dots. Quadrature amplitude variances and the Wigner function are employed to illustrate how the state of the boson mode can be controlled by a stationary electron current through the dots.
\end{abstract}

PACS numbers: 73.21.La,71.38.-k,62.25.+g,42.50.-p

\section{INTRODUCTION}

The two-level system coupled to a single bosonic mode (Rabi Hamiltonian ${ }^{1}$ ) is probably one of the best studied models for the interaction of matter with light ${ }^{2}$. Cavity quantum electrodynamics is an example where the coupling between atoms and photons can be studied in detail and used in order to, e.g., transfer quantum coherence from light to matter (control of tunneling by electromagnetic fields $\left.{ }^{3.4}\right)$ and vice versa ${ }^{5.6 .7}$.

Quantum optics usually deals with closed two-level systems where the total electron number on individual atoms remains constant and does not fluctuate during the interaction with the photon. This restriction can be lifted in semiconductor quantum dots (artificial atoms) by tunnel coupling to electron reservoirs. For example, in semiconductor cavity quantum electrodynamics 8 the interaction of light with excitons can be steered by 'pumping' the cavity by resonant tunneling of electrons and holes.

In this paper, we examine a single bosonic mode interacting with a system of two (bound) electronic states which are themselves coupled to a continuum of free electrons. As a concrete realization we investigate the stationary electron transport through a double quantum dot coupled to electron reservoirs and a single photon or phonon mode. The main idea is to control the density matrix of the coupled dot-boson system by external parameters such as the reservoir chemical potentials and the tunnel couplings. These parameters (which in experiments can be controlled via gate voltages) then determine the reduced density operators of the dot and the boson and allow one, e.g., to modify the state of the boson by driving a stationary electron current through the dots.

One of the motivations for our work are experiments in double quantum dots where the coherent coupling between classical light (microwaves) and electrons can be detected in electronic transport 9,10,11,12. The open twolevel-boson model describes the full quantum version of these systems with the light replaced by a photon field with its own quantum dynamics.

On the other hand, quantum effects (like spontaneous emission) relevant for transport through double dots have been found to be due to phonons rather than pho- tons. In fact, the importance of electron-phonon coupling for transport in coupled dots is well-established by now 13,14,15,16,17. Suggestions have been made ${ }^{14,18,19}$ to explore 'semiconductor phonon cavity QED' in nanostructures where phonons become experimentally controllable. In contrast to standard semiconductor cavity $\mathrm{QED}^{8}$, boundary conditions for vibration modes lead to extremely non-linear phonon dispersion relations. For example, van-Hove singularities in the phonon DOS show up at certain frequencies ${ }^{18}$, signatures of which seem to be relevant for transport through quantum dots embedded into free-standing structures ${ }^{20}$. Such a situation would then be described (within a strongly idealized model) by the coupling of a single frequency boson mode to a few-level quantum dot.

The influence of 'nano-mechanical' vibrational properties on single electron tunneling in fact has emerged as a whole new area of mesoscopic transport, triggered by the possibility to explore electron transport through individ-

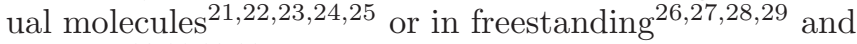
movable 30.31 .32 .33 nano-structures. Phonons then are no longer a mere source of dissipation but become experimentally controllable with the possibility, e.g., to create phonon confinement ${ }^{18.34 .35 .36}$ or the analogon of quantum optical phenomena such as coherent and squeezed phonon states $37,38,39,40$

The paper is organized as follows: in section III we introduce the model and derive a master equation for the density operator. We discuss transport properties in section III where a comparison to analytical solutions is made. In section IV the reduced boson density operator, its Wigner function and the fluctuation properties of the boson mode under a stationary electron current are discussed. Finally, we conclude with a discussion in section $\nabla$

\section{MODEL AND METHOD}

We consider an idealized situation of a single cavity boson mode (photon or phonon) coupled to a two-level electronic system (dot) which itself is connected to external electron reservoirs. Our aim is to determine the stationary reduced density operator $\rho(t)$ of the dot-boson 
system for large times $t$, treating the dot and the boson on equal footing. The single bosonic mode (photon or phonon) interacts with the electrons within the dots. We set $\hbar=1$ in the following.

\section{A. The Hamiltonian}

An artificial 'open' two-level system can be realized by two quantum dots (which for simplicity we call 'left' and 'right') coupled to each other by a tunnel barrier and to a 'left' source and 'right' drain electron reservoir with chemical potentials $\mu_{L}$ and $\mu_{R}$. In the following, we are only interested in the regime of high bias voltage $V=\mu_{L}-\mu_{R}>0$ We assume that the charging energy $U$ required to add an additional electron to the double dot is much greater than $V$ (strong Coulomb blockade regime). Therefore, electrons tunnel through the structure only from the left to the right. Only one additional electron can tunnel into the double dot at a time, and the effective Hilbert space of the electronic system can be defined by three states 10.16 'empty', 'left', and 'right', $|0\rangle=\left|N_{L}, N_{R}\right\rangle,|L\rangle=\left|N_{L}+1, N_{R}\right\rangle,|R\rangle=\left|N_{L}, N_{R}+1\right\rangle$. The energies of the two states with an additional electron in the left (right) dot are denoted as $\varepsilon_{L}\left(\varepsilon_{R}\right)$. Higher excited states for both dots are assumed to lie outside the energy window

$$
\mu_{L} \gg \varepsilon_{L}, \varepsilon_{R} \gg \mu_{R},
$$

which defines the regime where nonlinear transport does not significantly depend on $\mu_{L}$ and $\mu_{R}$ at low temperatures. This corresponds to the standard situation 10.14 .16 where only the two lowest hybridized electronic states in the double dot contribute to transport.

The Hamiltonian $H_{D}$ of the double dot is given in terms of the dot-operators $n_{L}:=|L\rangle\left\langle L\left|, n_{R}:=\right| R\right\rangle\langle R|$, and a term $H_{T}$ describing the tunneling between the left and the right dot,

$$
\begin{aligned}
H_{D} & =H_{A}+H_{T} \equiv \varepsilon_{L} n_{L}+\varepsilon_{R} n_{R}+T_{c}\left(P+P^{\dagger}\right), \\
P & =|L\rangle\left\langle R\left|, \quad P^{\dagger}=\right| R\right\rangle\langle L| .
\end{aligned}
$$

The tunnel matrix element $T_{c}$ is used to describe the strength of the tunneling process.

The Hilbert space of the bosonic system is spanned by the usual number or Fock eigenstates $|n\rangle, n=0,1,2,3, \ldots$ of the harmonic oscillator

$$
H_{p}=\omega a^{\dagger} a
$$

with frequency $\omega$ and creation/annihilation operators $a$ and $a^{\dagger}$ fulfilling $\left[a, a^{\dagger}\right]=1$. The coupling between the electronic and the bosonic system is described by four microscopic coupling constants,

$$
H_{e p}=\left(\alpha n_{L}+\beta n_{R}+\gamma^{*} P+\gamma P^{\dagger}\right)\left(a+a^{\dagger}\right) .
$$

Here, we assume the coupling constants $\alpha, \beta$, and $\gamma$ as given parameters. Their precise form can be calculated from microscopic details such as the many-body wave functions of the dot electrons. It should be noted that the interaction between the bosonic system and the reservoirs is not considered here.

The two electron reservoirs are described by

$$
H_{\text {res }}=\sum_{k} \varepsilon_{k}^{L} c_{k}^{\dagger} c_{k}+\sum_{k} \varepsilon_{k}^{R} d_{k}^{\dagger} d_{k},
$$

where the sum is over all wave vectors $k$ in both the left $(\mathrm{L})$ and right $(\mathrm{R})$ reservoirs and spin polarization is assumed for simplicity . The coupling between dot and reservoirs is described with two dot operators

$$
s_{L}=|0\rangle\left\langle L\left|, \quad s_{R}=\right| 0\right\rangle\langle R|
$$

and the tunnel Hamiltonian

$$
H_{V}=\sum_{k}\left(V_{k}^{L} c_{k}^{\dagger} s_{L}+V_{k}^{R} d_{k}^{\dagger} s_{R}+c . c\right)
$$

with tunnel matrix elements $V_{k}^{L}$ and $V_{k}^{R}$.

The total Hamiltonian is written as

$$
H=H_{0}+H_{T}+H_{V}+H_{e p},
$$

where $H_{0}=H_{A}+H_{p}+H_{\text {res }}$ is the free Hamiltonian in the interaction picture introduced below.

\section{B. Equations of Motion}

The density matrix of the total system (dot, boson and reservoirs) is given by the Liouville-von-Neumann equation and formulated in the interaction picture with respect to $H_{0}$. For the non-linear transport window Eq. (1) considered here, the chemical potentials in the equilibrium reservoirs are such that the Fermi distributions in the left and the right lead can well be approximated by $f_{L}(\varepsilon)=1$ and $f_{R}(\varepsilon)=0$. We treat the reservoir coupling term, $H_{V}$, in second order perturbation theory, neglecting Kondo physics ${ }^{41}$ throughout so that transport can be described by a master equation 10,16,42,43. Transforming back into the Schrödinger picture produces the following master equation for the reduced density operator of the system (dot + boson),

$$
\begin{aligned}
\frac{d}{d t} \rho(t) & =-i\left[H_{A}+H_{p}+H_{T}+H_{e p}, \rho(t)\right] \\
& -\frac{\Gamma_{L}}{2}\left(s_{L} s_{L}^{\dagger} \rho(t)-2 s_{L}^{\dagger} \rho(t) s_{L}+\rho(t) s_{L} s_{L}^{\dagger}\right) \\
& -\frac{\Gamma_{R}}{2}\left(s_{R}^{\dagger} s_{R} \rho(t)-2 s_{R} \rho(t) s_{R}^{\dagger}+\rho(t) s_{R}^{\dagger} s_{R}\right) \\
& -\frac{\gamma_{b}}{2}\left(2 a \rho a^{\dagger}-a^{\dagger} a \rho-\rho a^{\dagger} a\right)
\end{aligned}
$$

The perturbation from the reservoir is given by the tunnel rates

$$
\Gamma_{L / R}=2 \pi \sum_{k}\left|V_{k}^{L / R}\right|^{2} \delta\left(\varepsilon_{L / R}-\varepsilon_{k}^{L / R}\right) .
$$




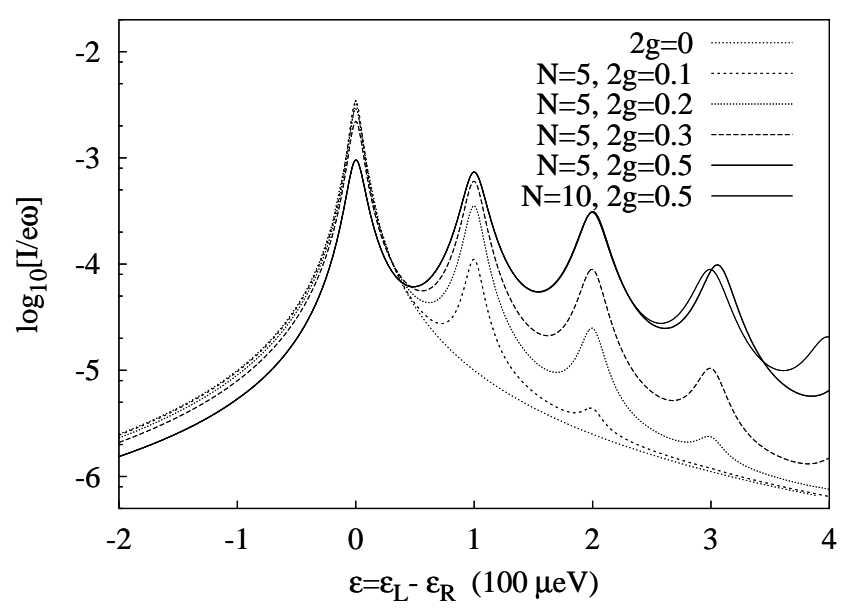

FIG. 1: Stationary current through double quantum dot coupled to single boson mode with $\Gamma_{L}=\Gamma_{R}=0.1, T_{c}=0.01$, $\gamma_{b}=0.05$ and varying $2 g=\alpha=-\beta$. (electron-boson coupling, cf. Eq. (4) ). $N$ : number of boson states.

In the last line in Eq. (9), we introduced a term that describes damping of the bosonic system at a rate $\gamma_{b} \underline{44}$ corresponding to photon or phonon cavity losses.

Taking matrix elements of the master equation, Eq. (9), one obtains a system of linear equations for the matrix elements of the density operator which are given explicitly in appendix $\mathrm{A}$ In the stationary state of these equations, the time derivatives are zero, and a coupled set of linear equations is found. In order to numerically solve these equations, the bosonic Hilbert space has to be truncated at a finite number $N$ of boson states. The total number of equations then is $5 N^{2}+10 N+5$, remembering that there is always an equation for $n=m=0$. The numerical solution becomes a standard matrix inversion, though book-keeping of the matrix elements has to be done carefully. The trace over electron and boson degrees of freedom has to to obey the normalization condition

$$
\begin{aligned}
\sum_{n}\left[\left\langle n, 0\left|\rho_{\text {stat }}\right| n, 0\right\rangle\right. & +\left\langle n, R\left|\rho_{\text {stat }}\right| n, R\right\rangle \\
& \left.+\left\langle n, L\left|\rho_{\text {stat }}\right| n, L\right\rangle\right]=1 .
\end{aligned}
$$

We mention that without this condition, the matrix becomes singular, and cannot be solved.

\section{TRANSPORT PROPERTIES}

The first quantity of interest that one can obtain from the matrix elements is the stationary electron current through the double dot.

\section{A. Stationary Current: Numerical Results}

In the stationary state, the current operator is defined via the rate of electrons tunneling from the left dot to the right dot,

$$
\hat{I}=\frac{\partial}{\partial t} n_{L}=i T_{C}\left(P^{\dagger}-P\right)+i\left(\gamma P^{\dagger}-\gamma^{*} P\right)\left(a+a^{\dagger}\right) .
$$

Here and in the following we set the electron charge $-e=$ 1 . For a boson mode with wave vector $\mathbf{Q}$, the electronboson coupling constants can be expressed in terms of matrix elements of the left and right dot states, $\alpha=$ $\lambda\left\langle L\left|e^{i \mathbf{Q r}}\right| L\right\rangle, \beta=\lambda\left\langle R\left|e^{i \mathbf{Q r}}\right| R\right\rangle, \gamma=\lambda\left\langle L\left|e^{i \mathbf{Q} \mathbf{r}}\right| R\right\rangle$, where $\lambda$ is the microscopic constant for the interaction of the boson with the electron in 2D.

Our formalism works for arbitrary choices of coupling parameters $\alpha, \beta, \gamma$, but for simplicity we restrict ourselves to simple, non-trivial cases for the numerical calculations. We set $\gamma=0$ which corresponds to neglecting non-diagonal terms that for relatively sharply peaked electron densities in the dots only weakly contribute 45 . For a sharp electron density profile, one furthermore has $\beta=\alpha e^{i \mathbf{Q d}}$ where $\mathbf{d}$ is the vector connecting the left and right dot centers. Identical energy shifts in both dots, corresponding to $\beta=\alpha$, have no effect on transport. In the following, we choose $\mathbf{Q d}=\pi$ and therefore electronboson coupling constants

$$
\alpha=-\beta \equiv 2 g, \quad \gamma=0 .
$$

Furthermore, the tunneling rates between the reservoirs and the dots are generally kept smaller than the energies of the dots and boson states. This produces clear and sharply defined transport characteristics. We use the boson frequency as an energy scale and set $\omega=1$ in the following. Using the typical phonon cavity energy $\hbar \omega \approx 100 \mu \mathrm{eV}$ from a recent experiment with a freestanding phonon cavity ${ }^{20}$ yields a typical electron current scale of $e \omega=24 \mathrm{nA}$ in Figs. 1 and 2

Fig. 1 illustrates the stationary current as a function of the energy difference $\varepsilon$. For zero boson coupling $g=0$, we reproduce the Lorentzian resonant tunneling profile

$$
I_{\text {stat }}=T_{c}^{2} \Gamma_{R} /\left[T_{c}^{2}\left(2+\Gamma_{R} / \Gamma_{L}\right)+\Gamma_{R}^{2} / 4+\varepsilon^{2}\right]
$$

as first derived by Stoof and Nazarov 10 .

For finite electron-boson coupling and positive $\varepsilon$, resonances appear when

$$
\varepsilon \equiv \varepsilon_{L}-\varepsilon_{R}=n \omega,
$$

i.e., the energy gap of the dots becomes equal to multiple integers of the boson energy. At these resonances, electrons tunneling from the left to the right dot can excite the boson system. Note that for larger $T_{c}, \varepsilon=\varepsilon_{L}-\varepsilon_{R}$ in Eq. (15) has to be replaced by the energy difference $\Delta \equiv \sqrt{\varepsilon^{2}+4 T_{c}^{2}}$ between bonding and anti-bonding state in the double dot. 


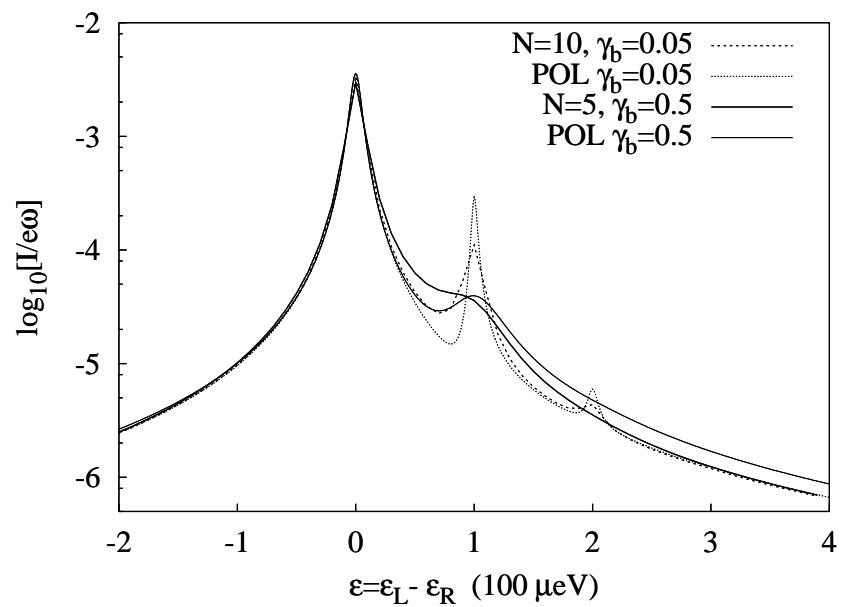

FIG. 2: Current as in Fig. 11 for coupling $2 g=0.1$ and different boson damping $\gamma_{b}$, alongside corresponding polaron transformation result, Eq. [19]

The current profiles with differing damping rates, Fig.(2), illustrate that the system is extremely sensitive even to very small 'damping'. For small but finite boson damping, we found that two profiles for $N=5$ and $N=10$ match extremely closely. This illustrates that a finite boson damping removes numerical problems due to the truncation of the boson Hilbert space at a finite $N$. As expected, there are no peaks at all on the absorption side of the profile $(\varepsilon<0)$ : the damped boson relaxes to its ground state.

\section{B. Perturbation Theory in $T_{c}$ : Polaron Transformation Method}

An analytical expression for the stationary current can be obtained from a polaron transformation and a polaron-transformed master equation that is valid for small coupling $T_{c}$ between the two dots ${ }^{16}$. After tracing out the bosonic degrees of freedom one obtains an equation of motion for the time-dependent expectation values of the operators $n_{L / R}$ and $P^{(\dagger)}$ which can be transformed into Laplace-space,

$$
\begin{aligned}
\left\langle n_{L}\right\rangle(z) & =-i \frac{T_{c}}{z}\left\{\langle P\rangle(z)-\left\langle P^{\dagger}\right\rangle(z)\right\} \\
& +\frac{\Gamma_{L}}{z}\left\{1 / z-\left\langle n_{L}\right\rangle(z)-\left\langle n_{R}\right\rangle(z)\right\} \\
\left\langle n_{R}\right\rangle(z) & =i \frac{T_{c}}{z}\left\{\langle P\rangle(z)-\left\langle P^{\dagger}\right\rangle(z)\right\}-\frac{\Gamma_{R}}{z}\left\langle n_{R}\right\rangle(z) \\
\langle P\rangle(z) & =-i T_{c}\left\{\left\langle n_{L}\right\rangle(z) C_{\varepsilon}(z)-\left\langle n_{R}\right\rangle(z) C_{-\varepsilon}^{*}(z)\right\} \\
& -\frac{\Gamma_{R}}{2}\langle P\rangle(z) C_{\varepsilon}(z) .
\end{aligned}
$$

Here, the coupling to the boson system enters through the correlation function

$$
\begin{aligned}
C_{\varepsilon}(z) & =\int_{0}^{\infty} d t e^{-z t+i \varepsilon t} C(t) \\
C(t) & =\left\langle X(t) X^{\dagger}(t=0)\right\rangle, \quad X=D\left(\frac{4 g}{\omega}\right)
\end{aligned}
$$

of the displacement operator of the bosonic mode,

$$
D(\xi)=\exp \left(\xi a^{\dagger}-\xi^{*} a\right)
$$

The equations (16) can be solved algebraically. One then obtains the expectation value $\langle I\rangle_{t \rightarrow \infty}$ of the stationary current from the $1 / z$-coefficient of the $I(z)$-expansion into a Laurent $\operatorname{series}^{46}$ for $z \rightarrow 0$,

$$
\begin{gathered}
\langle I\rangle_{t \rightarrow \infty}=T_{c}^{2} \frac{2 \Re\left(C_{\varepsilon}\right)+\Gamma_{R}\left|C_{\varepsilon}\right|^{2}}{\left|1+\frac{\Gamma_{R}}{2} C_{\varepsilon}\right|^{2}+2 T_{c}^{2} B_{\varepsilon}} \\
B_{\varepsilon}:=\Re\left\{\left(1+\frac{\Gamma_{R}}{2} C_{\varepsilon}\right)\left[\frac{C_{-\varepsilon}}{\Gamma_{R}}+\frac{C_{\varepsilon}^{*}}{\Gamma_{L}}\left(1+\frac{\Gamma_{L}}{\Gamma_{R}}\right)\right]\right\},
\end{gathered}
$$

where $C_{\varepsilon} \equiv C_{\varepsilon}(z \rightarrow 0)$.

The correlation function, $C(t)$, enters via a factorization assumption in the polaron-transformed master equation and has to be calculated from an effective density operator $\rho_{\mathrm{B}}$ of the bosonic mode. For dissipative pseudospin-boson systems with a continuous spectrum of modes and a thermal equilibrium for $\rho_{\mathrm{B}}$, reasonable results can be obtained within this method for small coupling, in particular in comparison with perturbation theory ${ }^{45,47}$. However, in the present case with just one single mode it is not clear whether or not a factorization into an effective dot and an effective boson density operator yields reasonable results, and in principle one should at least determine $\rho_{\mathrm{B}}$ self-consistently. For small couplings $g$, however, the assumption of a decoupled timeevolution of the boson should work well, and we proceed with a master equation for $\rho_{\mathrm{B}}$,

$$
\begin{aligned}
\frac{d}{d t} \rho_{\mathrm{B}}(t) & =-i\left[\omega a^{\dagger} a, \rho_{\mathrm{B}}\right] \\
& -\frac{\gamma_{b}}{2}\left(2 a \rho_{\mathrm{B}} a^{\dagger}-a^{\dagger} a \rho_{\mathrm{B}}-\rho_{\mathrm{B}} a^{\dagger} a\right)
\end{aligned}
$$

that describes the free time-evolution of the boson in presence of boson damping with damping constant $\gamma_{b}>$ 0 . Assuming the boson initial condition to be its ground state, one obtains

$$
C(t)=\exp \left\{-|\xi|^{2}\left(1-e^{-\left(\frac{\gamma_{b}}{2}+i \omega\right) t}\right)\right\}, \quad \xi=\frac{4 g}{\omega} .
$$

Fig. (2) shows that the analytical expression for the current, Eq. (19), compares quite well with the numerical results. In particular, this demonstrates that the formulation in terms of a general boson correlation function $C(t)$ works reasonably well not only for boson systems with continous mode spectrum ${ }^{16,45}$ but also for the single mode case discussed here, at least for small coupling constants $g$. We point out that the existence of a finite boson damping is crucial here and that for larger $g$, the comparison becomes worse, as expected. 


\section{BOSON DISTRIBUTION}

Turning now to properties of the bosonic system, our primary interest is how the boson mode can be controlled via parameters of the electron sub-system such as the bias $\varepsilon$ and the tunnel couplings $\Gamma_{R / L}$. In the stationary state, the reduced density operator of the boson is

$$
\rho_{b} \equiv \lim _{t \rightarrow \infty} \operatorname{Tr}_{\operatorname{dot}} \rho(t)=\lim _{t \rightarrow \infty} \sum_{i=0, L, R} \rho^{i i}(t) .
$$

\section{A. Boson state detection}

Before discussing the boson state $\rho_{b}$ we describe a possible experimental scheme to directly detect its properties. The basic idea is to use another nearby double quantum dot as detector of the boson, similar to the scheme for detecting quantum noise in mesoscopic conductors as proposed by Aguado and Kouwenhoven 48 .

The detector consists of a double quantum dot very similar to the one discussed above. The boson state gives a contribution to the inelastic, stationary current

$$
\begin{aligned}
I_{d}(E) \approx T_{d}^{2} P(E) & =2 T_{d}^{2} \Re \int_{0}^{\infty} d t e^{i E t} C_{d}(t) \\
C_{d}(t) & =\operatorname{Tr}\left[\rho_{b} X_{d}(t) X_{d}^{\dagger}\right], \quad X_{d}=D\left(\frac{4 g_{d}}{\omega}\right),
\end{aligned}
$$

through the detector in lowest order in the detector tunnel coupling $T_{d}$. Here, $E$ denotes the detector dot energy difference, $D$ again denotes the unitary displacement operator, Eq. [18), $g_{d}$ the coupling constant in the detector double dot, and $\rho_{b}$ the boson state. Eq. (23) describes the detector current $I(E)$ resulting from a given boson state $\rho_{b}$ as calculated in the next section.

Before discussing our numerical results for $\rho_{b}$, it is illustrative to look at a few special cases. For example, if the boson state $\rho_{b}$ describes a thermal equilibrium at temperature $T$, one obtains the inelastic current spectrum with absorption $E<0$ and emission $E>0$ branches ${ }^{16}$, as has been observed by Fujisawa et al ${ }^{14}$ for the case of equilibrium (multimode) phonons.

If $\rho_{b}=|n\rangle\langle n|$ describes a pure, $n$-boson number state, the function $C_{d}(t)$ in the detector current, Eq. (23), becomes $\left(\xi=4 g_{d} / \omega\right)$

$$
\begin{aligned}
C_{d}(t) & =\left\langle n\left|D\left(\xi e^{i \omega t}\right) D(-\xi)\right| n\right\rangle= \\
& =e^{-i|\xi|^{2} \sin \omega t} e^{-\frac{1}{2}\left|\xi\left(1-e^{i \omega t}\right)\right|^{2}} L_{n}^{0}\left(\left|\xi\left(1-e^{i \omega t}\right)\right|^{2}\right),
\end{aligned}
$$

where $L_{n}^{0}$ is a Laguerre polynomial, cf. appendix B Here we assume that the boson time-evolution is undamped in the detector, i.e. governed by the boson Hamiltonian $H_{b}=\omega a^{\dagger} a$. Expanding up to lowest order in $\xi=4 g_{d} / \omega$ and using $L_{n}^{0}(x)=1-n x+O\left(x^{2}\right)$, one obtains from Eq.(23) and Eq.(24)

$$
\begin{aligned}
I_{d}(E) & \approx T_{d}^{2} 2 \pi\left[\left\{1-|\xi|^{2}(2 n+1)\right\} \delta(E)\right. \\
& \left.+|\xi|^{2}\{n \delta(E+\omega)+(n+1) \delta(E-\omega)\}\right]
\end{aligned}
$$

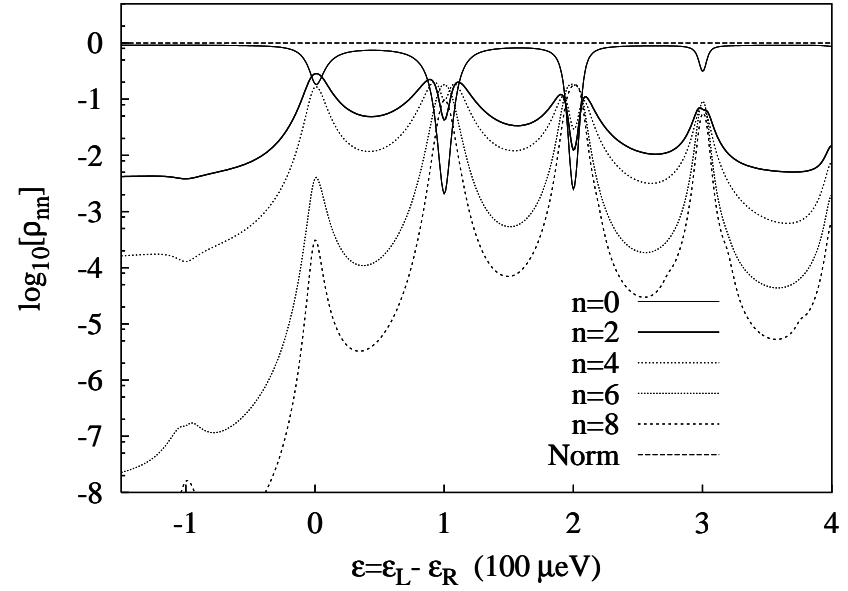

FIG. 3: Boson profiles $\mathrm{n}=0,2,4 \ldots 10$ for $\gamma_{b}=0.0001, N=10$, $2 g=\alpha=-\beta=0.3,10 \times T_{C}=\Gamma_{L}=\Gamma_{R}=0.1$.

Clearly, the height of the inelastic current peaks is determined by the quantum number $n$, i.e. the absorption peak at $E=-\omega<0$ scales with $n$, and the (stimulated) emission peak at $E=\omega>0$ scales with $n+1$.

The above example can be even generalised. In appendix $\mathrm{C}$ we show how to reconstruct an arbitrary boson state $\rho_{b}=\sum_{n m} \rho_{n m}|n\rangle\langle m|$ from the function $P(E)$ (or equivalently the stationary detector current spectrum $I_{d}(E)$, Eq. (23) ). This demonstrates that the properties of the boson state discussed in the following can be directly related to an experimentally accessible quantity.

\section{B. Numerical results}

We first analyse the boson mode via moments of its distribution function such as occupation number and Fano factor, before discussing the full distribution function in the Wigner function representation.

The boson occupation probability $p_{n}=\left(\rho_{b}\right)_{n n}$ is the probability for the boson to be in one particular number state $n$. Furthermore, the boson Fano factor $F^{Q}$ is calculated from the boson occupancy as the variance of boson number over the mean number of bosons,

$$
F^{Q}=\frac{\left\langle\hat{n}^{2}\right\rangle-\langle\hat{n}\rangle^{2}}{\langle\hat{n}\rangle} .
$$

Similarly, the variance $\Delta x^{2}=\left\langle x^{2}\right\rangle-\langle x\rangle^{2}$ and $\Delta p^{2}=$ $\left\langle p^{2}\right\rangle-\langle p\rangle^{2}$ of the position and a momentum coordinate

$$
x=\frac{\left(a+a^{\dagger}\right)}{\sqrt{2}}, \quad p=\frac{i\left(-a+a^{\dagger}\right)}{\sqrt{2}}
$$

can be obtained from $\rho_{b}$, cf. Eq. A7 in appendix A They provide an indication of the quantum fluctuations in the boson states, and prove useful in comparison with the Fano factor profile. It should also be apparent that $\Delta x^{2} \Delta p^{2} \geq \frac{1}{4}$. 


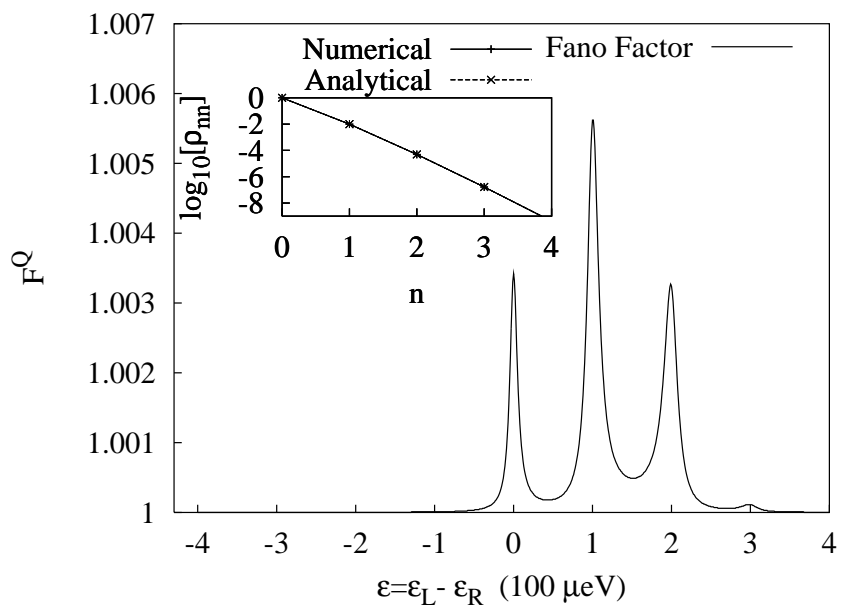

FIG. 4: Fano versus $\varepsilon$ profile for $2 g=\alpha=-\beta=0.1, N=10$, $\gamma_{b}=0.05,10 \times T_{C}=\Gamma_{L}=\Gamma_{R}=0.1$. Inset: Comparison between coherent state, Eq. 229], and numerical result for boson distribution $p_{n}$ at $\varepsilon=-4$.

We have checked that the probability $p_{n}$ fulfills the normalisation $\sum_{n} p_{n}=1$. For accurate results the occupation probability should tend to 0 as $n \rightarrow N$, where $N$ is the dimension of the truncated boson Hilbert space. This condition is easily achieved in presence of a finite boson damping rate, $\gamma_{b}>0$. In this case, it is possible to obtain a boson distribution which is centered around the lower boson number states, and is excited when a resonant interaction with the electron occurs.

It is more illustrative to first discuss a weakly damped case, as shown in Fig. 3 We can see quite clearly how at the positive resonance energies, the occupation probabilities of the states spreads into the higher number states.

The Fano factor $F^{Q}$ illustrates fluctuation properties of the boson mode with $F^{Q}=1$ corresponding to a Poissonian boson number distribution and $F^{Q}<1\left(F^{Q}>1\right)$ to a sub (super)-Poissonian distribution. Fig. 目illustrates the Fano factor vs. $\varepsilon$ for the strongly damped case.

This profile suggests the reduced boson states are coherent (Poissonian) for $\varepsilon<0$, and super-Poissonian at the resonance energies, $\varepsilon=\omega, 2 \omega, \ldots$.

\section{Coherent state and $x-p$ variances}

For $\varepsilon \ll 0$, nearly no current is flowing, the electron is predominatly localized in the left dot, and one can approximate the operator $\sigma_{z}=|L\rangle\langle L|-| R\rangle\langle R|$ by its expectation value $\left\langle\sigma_{z}\right\rangle=1$. Then, the boson system is effectively described by

$$
H_{\text {eff }}=2 g\left(a+a^{\dagger}\right)+\omega a^{\dagger} a
$$

which is a shifted oscillator, the ground state $|G S\rangle$ of which is the coherent state $|G S\rangle=|-2 g / \omega\rangle$ with $a|z\rangle=$ $z|z\rangle$. This can be seen by introducing new operators

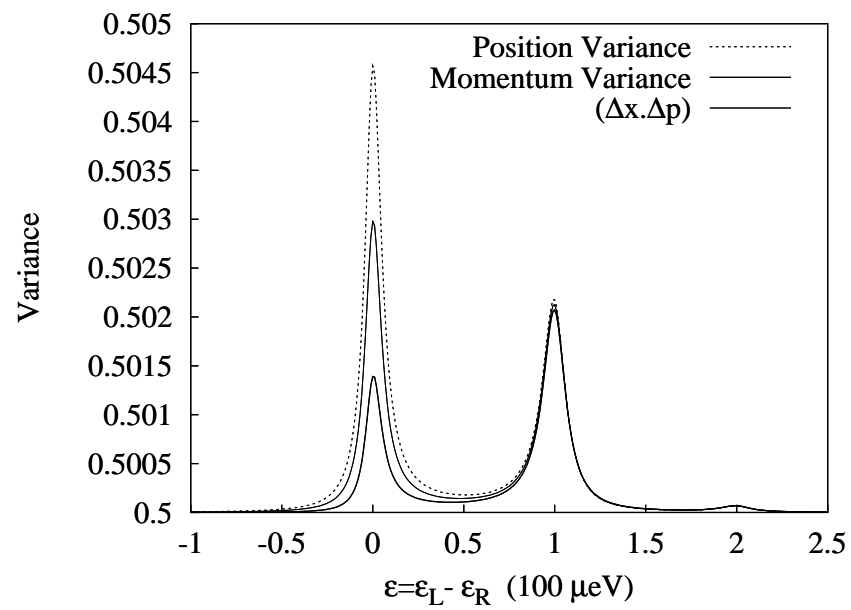

FIG. 5: Position variance, momentum variance, and an estimation of the combined uncertainty. $2 g=\alpha=-\beta=0.1$, $N=10, \gamma_{b}=0.05,10 \times T_{C}=\Gamma_{L}=\Gamma_{R}=0.1$.

$b:=a+2 g / \omega$ whence $H_{\text {eff }}=\omega b^{\dagger} b-4 g^{2} / \omega$ and $b|G S\rangle=0$. In this regime, we have

$$
\rho_{b} \approx|z\rangle\langle z|, \quad z=-2 g / \omega
$$

and $p_{n}=|\langle n \mid G S\rangle|^{2}$ is given by a Poisson distribution, $p_{n}=|z|^{2 n} e^{-|z|^{2}} / n$ !. One can plot this against the numerically obtained $p_{n}$ and check that the distribution is indeed Poissonian for small $\varepsilon \ll 0$. This is represented in the inset of Fig. 团in detail for the first 4 boson Fock (number) states.

The quantum fluctuations of the boson system is described by the variance of the position and momentum operators. Fig. 5 presents a direct comparison of the position and momentum variance of a strongly damped system. The uncertainty principle holds, $\Delta x \Delta p \geq \frac{1}{2}$, and thus $\Delta x^{2} \Delta p^{2} \geq \frac{1}{4}$. The state with minimum uncertainty occurs as expected, for $\varepsilon_{L}<\varepsilon_{R}$, with $\Delta x^{2} \Delta p^{2}=\frac{1}{4}$. This is a reassuring result, as it suggests both that the boson is coherent in this area, and that the Fano factor's Poissonian distribution for this coherent state is correct. We mention that since both $\Delta x>0.5$ and $\Delta p>0.5$, we find no squeezing of the boson mode for the parameters checked here.

\section{Wigner Function}

The Wigner function of the density operator $\rho_{b}$ for a bosonic mode $a^{\dagger}$ is a representation of $\rho_{b}$ in $x$ - $p$-space, cf. Eq.(27). It is defined as $\frac{49}{9}$

$$
W(x, p):=\frac{1}{\pi} \operatorname{Tr}\left(\rho_{b} D(2 \alpha) U_{0}\right), \quad \alpha=\frac{x+i p}{\sqrt{2}},
$$

where $D(\alpha):=\exp \left[\alpha a^{\dagger}-\alpha^{*} a\right]$, Eq.(18), is a unitary displacement operator and $U_{0}:=\exp \left[i \pi a^{\dagger} a\right]$ parity operator for the boson ${ }^{50}$. $W(x, p)$ is a symmetric Gaussian for 
$\varepsilon=0.00$

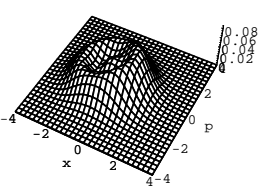

$\varepsilon=0.75$

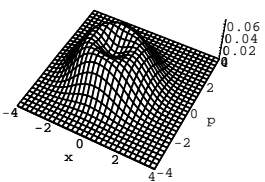

$\varepsilon=0.25$

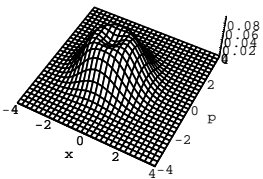

$\varepsilon=1.00$

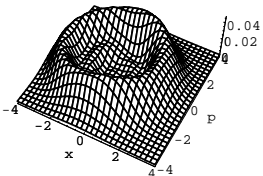

$\varepsilon=0.50$

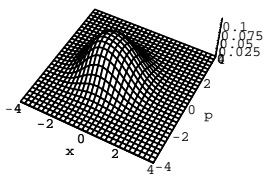

$\varepsilon=1.25$

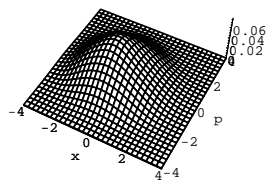

FIG. 6: Wigner distribution functions for the bosonic mode. Parameters are $\Gamma_{L}=\Gamma_{R}=T_{c}=0.1, \gamma_{b}=0.005, g=$ $0.2, N=20$. Stationary current resonances occur at $\varepsilon=$ $0.0,1.0,2.0, \ldots$.

a pure coherent boson state and a symmetric Gaussian multiplied with a polynomial for a pure number state $\underline{44}$. In our model, the shape of $W(x, p)$ therefore indicates how close to these limiting cases the actual stationary state $\rho_{b}$ of the boson, Eq.(22), is. In particular, this is a convenient way to represent the 'steering' of the boson mode when external parameters (like $\varepsilon$ or $\Gamma_{R / L}$ ) are changed.

Using the Fock state basis $\{|n\rangle, n=0,1,2, \ldots\}$ and $U_{0}=\sum_{n=0}^{\infty}(-1)^{n}|n\rangle\langle n|$, we find

$$
W(x, p)=\frac{1}{\pi} \sum_{n, m=0}^{\infty}(-1)^{n}\left\langle n\left|\rho_{b}\right| m\right\rangle\langle m|D(2 \alpha)| n\rangle .
$$

It is useful to split the sum into diagonal and nondiagonal parts, to use $\left\langle m\left|\rho_{b}\right| n\right\rangle=\left\langle n\left|\rho_{b}\right| m\right\rangle^{*}$ and $(m \geq n)$

$$
\begin{aligned}
\langle m|D(\alpha)| n\rangle & =\sqrt{\frac{n !}{m !}} \alpha^{m-n} e^{-\frac{1}{2}|\alpha|^{2}} L_{n}^{m-n}\left(|\alpha|^{2}\right) \\
& =\left\langle m\left|D^{\dagger}(\alpha)\right| n\right\rangle^{*}=(-1)^{m-n}\langle m|D(\alpha)| n\rangle^{*},
\end{aligned}
$$

where again $\alpha=(x+i p) / \sqrt{2}$ and $L_{n}^{m-n}$ is a Laguerre polynomial, cf. appendix B This leads to

$$
\begin{aligned}
& W(x, p)=\frac{1}{\pi} \sum_{n=0}^{\infty}(-1)^{n}\left\langle n\left|\rho_{b}\right| n\right\rangle\langle n|D(2 \alpha)| n\rangle \\
& +\frac{1}{\pi} \sum_{n=0}^{\infty} \sum_{m=n+1}^{\infty}(-1)^{n} 2 \operatorname{Re}\left[\left\langle n\left|\rho_{b}\right| m\right\rangle\langle m|D(2 \alpha)| n\rangle\right] .
\end{aligned}
$$

Using our numerical results for the stationary state $\rho$ of the coupled system, we easily obtain Wigner functions from Eq. (22) and Eq. (33) for different values of the energy difference $\varepsilon$ between the two dots as shown in Fig. (6). Between two resonance energies $\varepsilon=n \omega$, $W(x, p)$ closely resembles a Gaussian. At and close to the resonance energies, the distribution spreads out in rings around the origin, which is consistent with the increased Fock state occupation numbers and the increased position and momentum variances at these energies.

\section{DISCUSSION}

Our investigation is based on a numerical method which allows the density matrix of the boson-dot system to be solved for arbitrary dot parameters $\left(\varepsilon, T_{c}, \Gamma_{L / R}\right)$ and boson damping rate $\gamma_{b}$. Since we have to truncate the boson Hilbert space, the electron-boson coupling constant $g$ has to be restricted to small values $g \lesssim 1$. In contrast to the determination of the (pure) eigenstates of an isolated dot-boson system (Rabi-Hamiltonian), the numerical effort for our mixed state (density operator) is much bigger here. Although not discussed in this paper, we suggest that the strong coupling regime could be reached numerically by a polaron transformation of the master equation ${ }^{16}$ without the factorization assumption employed in section IIIB Fortunately, at present the small coupling regime seems to be valid for experimental situations with quantum dots $13,14,15,16$.

Our results suggest that there is no resonant interaction on the $\varepsilon<0$ side of the current peak as long as the boson system is damped and any many-body excited electron states can be ignored. For $\varepsilon>0$ we have found strong excitations of the boson mode occuring at resonances given by multiples $n \omega$ of the boson frequency $\omega$. These correspond to the photo-satellite peaks in resonant tunneling in electromagnetic $\mathrm{AC}$ fields ${ }^{9.10}$ which in the classical case, however, appear on both sides of the main resonance $n=0$ in contrast to the quantum case considered here. The resonances at $\varepsilon=n \omega$ can thus be interpreted as the emission of phonons (photons) by the electron as it tunnels through the dot. It should be possible to detect them in the stationary current through double quantum dots in 'phonon resonators'. With increasing electron-boson coupling, the visibility of these side peaks increases whereas the main resonance at $\varepsilon=0$ is reduced.

We have discussed that an additional double dot can serve as a detector of the stationary boson state $\rho_{b}$, if its energy difference $E$ is tuned independently. In the single mode case considered here, the stationary detector current spectrum $I_{d}(E)$ is uniquely related to the components of $\rho_{b}$ in the number state basis. In order to characterize the stationary boson state itself, we have investigated the Fano factor and the quantum fluctuations in the quadrature amplitudes of the boson. For $\varepsilon \ll 0$ the boson state is perfectly described as a coherent state whereas for $\varepsilon \gtrsim 0$ the interaction with the quantum dot produces occupied excited states in the boson mode that could best be visualized by a broadening of the phase space (Wigner) distribution function of the boson.

Although in this paper we have only calculated stationary properties, our method can be extended to calculate the noise-spectrum via the quantum regression theorem within the master equation framework as well ${ }^{44}$. It remains a task for the future to analyze the relation between the boson quantum fluctuations and the current noise in detail. 


\section{Acknowledgments}

We acknowledge inspiring discussions with A. Vourdas (who suggested the analysis of the $x$ and $p$ variances) and C. Emary. This work was supported by projects EPSRC GR44690/01, DFG Br1528/4-1, the WE Heraeus foundation and the UK Quantum Circuits Network.

\section{APPENDIX A: MATRIX ELEMENTS OF THE DENSITY OPERATOR}

The matrix elements of the dot-boson density operator $\rho$ are defined as

$$
\begin{aligned}
& \rho_{n m}^{i}:=\langle n, i|\rho| i, m\rangle, \quad i=0, L, R \\
& \rho_{n m}^{i j}:=\langle n, i|\rho| j, m\rangle, \quad i, j=0, L, R,
\end{aligned}
$$

where $n$ and $m$ refer to boson Fock states.

The matrix elements for the empty dot state obey

$$
\begin{aligned}
\frac{d}{d t} \rho_{n m}^{0} & =\left[i \omega(m-n) \rho_{n m}^{0}\right]-\Gamma_{L}\left[\rho_{n m}^{0}\right]+\Gamma_{R}\left[\rho_{n m}^{R}\right] \quad(\mathrm{A} 2) \\
& +\frac{\gamma_{b}}{2}\left[2 \rho_{n+1, m+1}^{0} \sqrt{n+1} \sqrt{m+1}-\rho_{n m}^{0}(n+m)\right]
\end{aligned}
$$

those for the right dot state

$$
\begin{aligned}
\frac{d}{d t} \rho_{n m}^{R} & =i\left[\omega(m-n) \rho_{n m}^{R}+T_{c}\left(\rho_{n m}^{R L}-\rho_{n m}^{L R}\right)\right. \\
& +\beta\left(\sqrt{m} \rho_{n m-1}^{R}+\sqrt{m+1} \rho_{n m+1}^{R}\right. \\
& \left.-\sqrt{n} \rho_{n-1 m}^{R}-\sqrt{n+1} \rho_{n+1 m}^{R}\right) \\
& +\gamma^{*}\left(\sqrt{m} \rho_{n m-1}^{R L}+\sqrt{m+1} \rho_{n m+1}^{R L}\right) \\
& \left.-\gamma\left(\sqrt{n} \rho_{n-1 m}^{L R}+\sqrt{n+1} \rho_{n+1 m}^{L R}\right)\right]-\Gamma_{R}\left[\rho_{n m}^{R}\right] \\
& +\frac{\gamma_{b}}{2}\left[2 \rho_{n+1, m+1}^{R} \sqrt{n+1} \sqrt{m+1}-\rho_{n m}^{R}(n+m)\right]
\end{aligned}
$$

and for the left dot state

$$
\begin{aligned}
\frac{d}{d t} \rho_{n m}^{L} & =i\left[\omega(m-n) \rho_{n m}^{L}+T_{c}\left(-\rho_{n m}^{R L}+\rho_{n m}^{L R}\right)\right. \\
& +\alpha\left(-\sqrt{n+1} \rho_{n+1 m}^{L}-\sqrt{n} \rho_{n-1 m}^{L}\right. \\
& \left.+\sqrt{m} \rho_{n m-1}^{L}+\sqrt{m+1} \rho_{n m+1}^{L}\right) \\
& +\gamma^{*}\left(-\sqrt{n+1} \rho_{n+1 m}^{R}-\sqrt{n} \rho_{n-1 m}^{R L}\right) \\
& \left.+\gamma\left(\sqrt{m} \rho_{n m-1}^{L R}+\sqrt{m+1} \rho_{n m+1}^{L R}\right)\right]+\Gamma_{L}\left[\rho_{n m}^{0}\right] \\
& +\frac{\gamma_{b}}{2}\left[2 \rho_{n+1, m+1}^{L} \sqrt{n+1} \sqrt{m+1}-\rho_{n m}^{L}(n+m)\right] .
\end{aligned}
$$

The equation of motion for the off-diagonal elements $\rho_{n m}^{R L}$ is

$$
\begin{aligned}
\frac{d}{d t} \rho_{n m}^{R L} & =i\left[\rho_{n m}^{R L}\left[\omega(m-n)+\left(\varepsilon_{L}-\varepsilon_{R}\right)\right]\right. \\
& +\beta\left(-\sqrt{n+1} \rho_{n+1 m}^{R L}-\sqrt{n} \rho_{n-1 m}^{R L}\right) \\
& +\alpha\left(\sqrt{m+1} \rho_{n m+1}^{R L}+\sqrt{m} \rho_{n m-1}^{R L}\right) \\
& +\gamma\left(-\sqrt{n+1} \rho_{n+1 m}^{L L}-\sqrt{n} \rho_{n-1 m}^{L L}\right. \\
& \left.+\sqrt{m+1} \rho_{n m+1}^{R R}+\sqrt{m} \rho_{n m-1}^{R R}\right) \\
& \left.+T_{c}\left(\rho_{n m}^{R R}-\rho_{n m}^{L L}\right)\right]-\frac{\Gamma_{R}}{2}\left[\rho_{n m}^{R L}\right] \\
& +\frac{\gamma_{b}}{2}\left[2 \rho_{n+1, m+1}^{R L} \sqrt{n+1} \sqrt{m+1}-\rho_{n m}^{R L}(n+m)\right] .
\end{aligned}
$$

Taking the trace over all the boson and electron states, the expression Eq. (12) for the electron current operator reads

$$
\begin{aligned}
\langle\hat{I}\rangle & =\sum_{n} i T_{C}\left[\rho_{n, n}^{L R}-\rho_{n, n}^{R L}\right] \\
& +\sum_{n} i\left[\gamma\left(\rho_{n, n-1}^{L R} \sqrt{n}+\rho_{n, n+1}^{L R} \sqrt{n+1}\right)\right. \\
& \left.-\gamma^{*}\left(\rho_{n, n-1}^{R L} \sqrt{n}+\rho_{n, n+1}^{R L} \sqrt{n+1}\right)\right] .
\end{aligned}
$$

The variances of the boson position and momentum coordinate, Eq. (27), are obtained by performing the trace over the dot variables $0, L, R$,

$$
\begin{aligned}
\Delta x^{2} & =\frac{1}{2} \operatorname{Tr}_{\operatorname{dot}} \sum_{n}\left(\sqrt{n} \sqrt{n-1} \rho_{n, n-2}\right. \\
& \left.+(2 n+1) \rho_{n, n}+\sqrt{n+1} \sqrt{n+2} \rho_{n, n+2}\right) \\
& -\frac{1}{2}\left[\operatorname{Tr}_{\operatorname{dot}} \sum_{n}\left[\sqrt{n} \rho_{n, n-1}+\sqrt{n+1} \rho_{n, n+1}\right]\right]^{2}, \\
\Delta p^{2} & =\frac{1}{2} \operatorname{Tr}_{\operatorname{dot}} \sum_{n}\left(-\sqrt{n} \sqrt{n-1} \rho_{n, n-2}\right. \\
& \left.+(2 n+1) \rho_{n, n}-\sqrt{n+1} \sqrt{n+2} \rho_{n, n+2}\right) \\
& -\frac{1}{2}\left[\operatorname{Tr}_{\operatorname{dot}} \sum_{n}\left[-i \sqrt{n} \rho_{n, n-1}+i \sqrt{n+1} \rho_{n, n+1}\right]\right]^{2} .
\end{aligned}
$$

\section{APPENDIX B: DISPLACEMENT OPERATOR, COHERENT STATES}

Here, we summarize some useful properties of the unitary displacement operator

$$
\begin{aligned}
D(z) & \equiv e^{z a^{\dagger}-z^{*} a}=\left(D^{\dagger}(z)\right)^{-1}=D^{\dagger}(-z) \\
& =e^{-\frac{1}{2}|z|^{2}} e^{z a^{\dagger}} e^{-z^{*} a}=e^{\frac{1}{2}|z|^{2}} e^{-z^{*} a} e^{z a^{\dagger}},
\end{aligned}
$$

where $z$ is a complex number and we used the operator exponential $e^{A+B}=e^{A} e^{B} e^{-(1 / 2)[A, B]}$ for $[[A, B], A]=$ $[[A, B], B]=0$, cf. also 44 . A coherent boson state $|z\rangle$ is defined as eigenstate of the annihilation operator, $a|z\rangle=$ $z|z\rangle$, where $z$ is a complex number. It can be generated from the boson vacuum $|0\rangle$ as

$$
|z\rangle=D(z)|0\rangle
$$


Extremely useful is the relation

$$
D(\alpha+\beta)=D(\alpha) D(\beta) e^{-i \Im\left(\alpha \beta^{*}\right)}
$$

for arbitrary complex numbers $\alpha, \beta$. Coherent state matrix elements of $D(\alpha)$ follow as

$$
\langle\beta|D(\alpha)| \beta\rangle=e^{-\frac{1}{2}|\alpha|^{2}} e^{2 i \Im\left(\alpha \beta^{*}\right)} .
$$

Number state matrix elements can be obtained using $|n\rangle \equiv(1 / \sqrt{n !})\left(a^{\dagger}\right)^{n}|0\rangle$,

$$
\begin{gathered}
\langle m|D(\alpha)| n\rangle=e^{\frac{1}{2}|\alpha|^{2}} \times \\
\times\left.\frac{1}{\sqrt{n ! m !}} \frac{\partial^{n+m}}{\partial z_{1}^{m} \partial z_{2}^{n}}\left\langle 0\left|e^{\left(z_{1}-\alpha^{*}\right) a} e^{\left(z_{2}+\alpha\right) a^{\dagger}}\right| 0\right\rangle\right|_{z_{1}=z_{2}=0} .
\end{gathered}
$$

With $\left\langle 0\left|e^{\left(z_{1}-\alpha^{*}\right) a} e^{\left(z_{2}+\alpha\right) a^{\dagger}}\right| 0\right\rangle=e^{\left(z_{1}-\alpha^{*}\right)\left(z_{2}+\alpha\right)}$, for $m \geq$ $n$ the differentiation yields

$$
\begin{aligned}
\langle m|D(\alpha)| n\rangle & =e^{\frac{1}{2}|\alpha|^{2}} \frac{1}{\sqrt{n ! m !}} \times \\
& \times\left.\frac{\partial^{n}}{\partial z_{2}^{n}}\left(z_{2}+\alpha\right)^{m} e^{-\left(z_{2}+\alpha\right) \alpha^{*}}\right|_{z_{2}+\alpha}
\end{aligned}
$$

Comparison with the generating function of the Laguerre polynomials $\frac{51}{5}$ yields Eq.(32). A corresponding expression can be derived for $n \geq m$.

\section{APPENDIX C: RELATION BETWEEN $P(E)$ AND STATIONARY BOSON STATE $\rho_{b}$}

Again we assume that the boson time-evolution is undamped in the detector, i.e. governed by the boson Hamiltonian $H_{b}=\omega a^{\dagger} a$. Expressing an arbitrary boson state $\rho_{b}$ in the number state basis, $\rho_{b}=\sum_{n m} \rho_{n m}|n\rangle\langle m|$,
Fourier transforming the function $P(E)$, Eq.(23), yields $\left(\xi=4 g_{d} / \omega\right)$

$$
\begin{aligned}
\tilde{P}(t) & \equiv \frac{1}{2 \pi} \int_{-\infty}^{\infty} d E e^{-i E t} P(E)=C_{d}(t)+C_{d}^{*}(-t) \\
& =\sum_{n m} \rho_{n m} c_{n m}(t) \\
c_{n m}(t) & \equiv\left\langle m\left|D\left(\xi e^{i \omega t}\right) D(-\xi)\right| n\right\rangle+\left\langle m\left|D(\xi) D\left(-\xi e^{-i \omega t}\right)\right| n\right\rangle .
\end{aligned}
$$

The functions $c_{n m}(t)$ can be calculated analytically, using Eq. (B4) and the matrix elements $\langle m|D(z)| n\rangle$ of the unitary displacement operator, Eq. (32). They are periodic in $2 \pi / \omega$, and from integrating Eq. (C1) over one period one obtains a linear relation between the Fourier coefficients $\tilde{P}^{k}$ and $c_{n m}^{k}$,

$$
\begin{aligned}
\tilde{P}^{k} & =\sum_{n m} c_{n m}^{k} \rho_{n m} \\
\tilde{P}^{k} & \equiv \frac{\omega}{2 \pi} \int_{0}^{\frac{2 \pi}{\omega}} \tilde{P}(t) e^{i k \omega t} d t \\
c_{n m}^{k} & \equiv \frac{\omega}{2 \pi} \int_{0}^{\frac{2 \pi}{\omega}} c_{n m}(t) e^{i k \omega t} d t .
\end{aligned}
$$

The $\tilde{P}^{k}$ have to be determined by numerical integration from the (experimentally given) $P(E)$. Regarding $(n m)$ as a single index, Eq. $\mathrm{C2}$ is a linear equation that can then be solved for the coefficients $\rho_{n m}$ of the boson state by inverting the matrix $c_{n m}^{k}$, the coefficients of which are given as Fourier coefficients of known expressions. In practical terms, the number of boson states as well as the number of Fourier coefficients have to be restricted in order to make this inversion feasable.

Eq. C22 establishes the relation between $P(E)$ (or, via Eq. (23), the detector current $I_{d}=T_{d}^{2} P(E)$ in lowest order $\left.T_{d}\right)$ and an arbitrary single mode boson state $\rho_{b}$.
1 I. I. Rabi, Phys. Rev. 51, 652 (1937).

${ }^{2}$ L. Allen and J. H. Eberly, Optical Resonance and TwoLevel Atoms (Dover, New York, 1987).

3 P. Neu and R. J. Silbey, Phys. Rev. A 54, 5323 (1996).

${ }^{4}$ M. Grifoni and P. Hänggi, Phys. Rep. 304, 229 (1998).

${ }^{5}$ M. Ueda, T. Wakabayashi, and M. Kuwata-Gonokami, Phys. Rev. Lett. 76, 2045 (1996).

${ }^{6}$ H. Saito and M. Ueda, Phys. Rev. Lett. 79, 3869 (1997).

7 H. Saito and M. Ueda, Phys. Rev. A 59, 3959 (1999).

8 Y. Yamamoto, F. Tassone, and H. Cao, Semiconductor Cavity Quantum Electrodynamics, Vol. 169 of Springer Tracts in Modern Physics (Springer, Berlin, 2000).

9 T. H. Oosterkamp, T. Fujisawa, W. G. van der Wiel, K. Ishibashi, R. V. Hijman, S. Tarucha, and L. P. Kouwenhoven, Nature 395, 873 (1998).

10 T. H. Stoof and Yu. V. Nazarov, Phys. Rev. B 53, 1050 (1996).

11 R. H. Blick, D. Pfannkuche, R. J. Haug, K. v. Klitzing, and K. Eberl, Phys. Rev. Lett. 80, 4032 (1998).

12 R. H. Blick, D. W. van der Weide, R. J. Haug, and
K. Eberl, Phys. Rev. Lett. 81, 689 (1998).

13 S. Tarucha, T. Fujisawa, K. Ono, D. G. Austin, T. H. Oosterkamp, W. G. van der Wiel, Microelectr. Engineer. 47, 101 (1999).

14 T. Fujisawa, T. H. Oosterkamp, W. G. van der Wiel, B. W. Broer, R. Aguado, S. Tarucha, and L. P. Kouwenhoven, Science 282, 932 (1998).

15 H. Qin, F. Simmel, R. H. Blick, J. P. Kotthaus, W. Wegscheider, M. Bichler, Phys. Rev. B 63, 035320 (2001).

16 T. Brandes and B. Kramer, Phys. Rev. Lett. 83, 3021 (1999).

17 T. Fujisawa, D. G. Austing, Y. Tokura, Y. Hirayama, and S. Tarucha, Nature 419, 278 (2002).

18 S. Debald, T. Brandes, B. Kramer, Phys. Rev. B (Rapid Comm.) 66, 041301(R) (2002).

19 I. Wilson-Rae and A. Imamoglu, Phys. Rev. B 65, 235311 (2002).

20 E. M. Höhberger, J. Kirschbaum, R. H. Blick, T. Brandes, W. Wegscheider, M. Bichler, and J. P. Kotthaus (unpub- 
lished), 2002

${ }^{21}$ H. Park, J. Park, A. K. L. Lim, E. H. Anderson, A. P. Alivisatos, and P. L. McEuen, Nature 407, 57 (2000).

22 C. Joachim, J. K. Gimzewski, A. Aviram, Nature 408, 541 (2000).

23 Reichert, R. Ochs, D. Beckmann, H. B. Weber, M. Mayor, and H. v. Löhneysen, Phys. Rev. Lett. 88, 176804 (2002).

24 D. Boese and H. Schoeller, Europhys. Lett. 54, 668 (2001).

25 A. O. Gogolin and A. Komnik, cond-mat/0207513 2002.

${ }^{26}$ A. N. Cleland, M. L. Roukes, Nature 392, 160 (1998).

27 R. H. Blick, F. G. Monzon, W. Wegscheider, M. Bichler, F. Stern, and M. L. Roukes, Phys. Rev. B 62, 17103 (2000).

28 N. F. Schwabe, A. N. Cleland, M. C. Cross, and M. I. Roukes, Phys. Rev. B 52, 12911 (1995).

${ }^{29}$ R. H. Blick, M. L. Roukes, W. Wegscheider, M. Bichler, Physica B 249, 784 (1998).

30 A. D. Armour and A. MacKinnon, Phys. Rev. B 66, 035333 (2002).

31 L. Y. Gorelik, A. Isacsson, M. V. Voinova, B. Kasemo, R. I. Shekhter, and M. Jonson, Phys. Rev. Lett. 80, 4526 (1998).

32 C. Weiss and W. Zwerger, Europhys. Lett. 47, 97 (1999).

33 A. Erbe, C. Weiss, W. Zwerger, and R. H. Blick, Phys. Rev. Lett. 87, 096106 (2001).

${ }^{34}$ N. Bannov, V. Mitin, M. Stroscio, phys. stat. sol. (b) 183, 131 (1994).

35 N. Bannov, V. Aristov, V. Mitin, M. A. Stroscio, Phys. Rev. B 51, 9930 (1995).

36 N. Nishiguchi, Y. Ando, M. N. Wybourne, J. Phys.: Conden. Matter 9, 5751 (1997).

37 M. P. Blencowe, Phys. Rev. B 59, 4992 (1999).
38 T. D. Kraus, F. W. Wise, Phys. Rev. Lett. 25, 5102 (1998).

39 X. Hu, F. Nori, Phys. Rev. Lett. 79, 4605 (1997).

40 M. P. Blencowe, M. N. Wybourne, Physica B 280, 555 (2000).

41 U. Hartmann and F. K. Wilhelm, phys. stat. sol. (b) 233, 385 (2002); U. Hartmann and F. K. Wilhelm, cond-mat/0212063

42 S. A. Gurvitz and Ya. S. Prager, Phys. Rev. B 53, 15932 (1996).

43 S. A. Gurvitz, Phys. Rev. B 57, 6602 (1998).

44 D. F. Walls and G. J. Milburn, Quantum Optics (Springer, Berlin, 1994).

45 T. Brandes, T. Vorrath, in Recent Progress in Many Body Physics, Advances in Quantum Many Body Theory, edited by R. Bishop, T. Brandes, K. Gernoth, N. Walet, and Y. Xian (World Scientific, Singapore, 2001).

46 G. Doetsch, Anleitung zum praktischen Gebrauch der Laplace-Transformation und der Z-Transformation (Oldenbourg, München, 1989).

47 T. Brandes and T. Vorrath, Phys. Rev. B 66, 075341 (2002).

48 R. Aguado and L. Kouwenhoven, Phys. Rev. Lett. 84, 1986 (2000).

49 K. E. Cahill and R. J. Glauber, Phys. Rev. 177, 1882 (1969).

50 S. Chountasis and A. Vourdas, Phys. Rev. A 58, 848 (1998).

51 I. S. Gradshteyn and I. M. Ryzhik, Table of Integrals, Series, and Products, 5 ed. (Academic Press, New York, 1994). 